\title{
Dispersão larval pós-alimentar de Lucilia sericata (Diptera, Calliphoridae) em condições de laboratório
}

\author{
Sabrina M. Pires ${ }^{1}$, Cristine R. Zimmer², Marcial C. Cárcamo ${ }^{1} \&$ Paulo B. Ribeiro ${ }^{1,2}$
}

\begin{abstract}
1. Programa de Pós-Graduação em Parasitologia, Laboratório de Biologia de Insetos, Departamento de Microbiologia e Parasitologia, Instituto de Biologia, Universidade Federal de Pelotas, Caixa Postal 354, 96010-900 Capão do Leão, RS, Brasil. (medpires@gmail.com; marcial.carcamo@gmail.com; bretanha@ufpel.tche.com.br)

2. Programa de Pós-Graduação em Fitossanidade, Departamento de Fitossanidade, Faculdade de Agronomia "Eliseu Maciel", Universidade Federal de Pelotas, Caixa Postal 354, 96010-900 Capão do Leão, RS, Brasil. (crzimmerbio@yahoo.com.br)
\end{abstract}

\begin{abstract}
Post-feeding larval dispersion of Lucilia sericata (Diptera, Calliphoridae) in laboratory. Calliphorid flies are the first organisms of the faunal succession associated with decomposing bodies, and can help in the estimation of post-mortem interval. Therefore, studies on post-feeding larval dispersion are relevant to medico-criminal investigations. A circular arena simulating the natural environment was built in the laboratory in order to examine the radial post-feeding larval dispersion of Lucilia sericata (Meigen, 1826) and the relationship between weight, distance, and depth of burial. Our findings indicated that most pupae were recovered at a depth between $6 \mathrm{~cm}$ and $8 \mathrm{~cm}$ and at a distance of $33 \mathrm{~cm}$ and $45 \mathrm{~cm}$ from the center of the arena. No significant differences were found regarding the propagation and depth of males, females, and non-viable individuals. The mean weight of females $(\mathrm{x}=32.35 \mathrm{mg})$ was higher than that of males $(\mathrm{x}=30.28 \mathrm{mg})$. The correlation and regression analysis between weight and distance, and between weight and depth was directly proportional suggesting that heavier pupae dispersed farther and deeper. Our study also showed that a circular arena allows the dispersion of larvae into all directions.
\end{abstract}

KEYWORDS. Forensic entomology, blowflies, legal medicine.

RESUMO. Os dípteros califorídeos são os primeiros indivíduos a compor a sucessão faunística de cadáveres em decomposição, auxiliando assim na estimativa do intervalo pós-morte. Estudos de dispersão pós-alimentar de suas larvas possuem relevância para investigações médico-criminais. Diante disto, uma arena circular, simulando o ambiente natural, foi montada em laboratório a fim de verificar-se a dispersão larval radial pós-alimentar de Lucilia sericata (Meigen, 1826), tentando assim estabelecer relações entre as variáveis peso, distância e profundidade de enterramento na arena. Os resultados demonstraram que $45 \%$ das pupas foram recuperadas a uma profundidade compreendida entre $6 \mathrm{~cm}$ e $8 \mathrm{~cm}$ e a uma distância entre $33 \mathrm{~cm}$ e $45 \mathrm{~cm}$ do centro da arena. Não houve diferenças significativas quanto à propagação e profundidade de enterramento de machos, fêmeas e indivíduos inviáveis. Com relação ao peso, verificou-se que a média das fêmeas $(x=32,35 \mathrm{mg})$ foi superior a dos machos $(\mathrm{x}=30,28 \mathrm{mg})$. A análise de correlação e de regressão entre peso e distância percorrida e entre peso e profundidade foram positivas, ou seja, pupas oriundas de larvas mais pesadas propagaram e se aprofundaram mais. O experimento permitiu ainda concluir que uma arena circular possibilita o deslocamento das larvas em todas as direções.

PALAVRAS-CHAVE. Entomologia forense, varejeiras, medicina legal.

Lucilia sericata (Meigen, 1826), é um califorídeo que apresenta caráter sinantrópico, sendo comumente encontrada ao redor de habitações humanas (GRASSBERGER \& REITER, 2001). Já foi registrado causando miíases nos olhos e nos seios paranasais de paciente em hospital (Daniel et al., 1994). Embora as espécies de Lucilia Robineau-Desvoidy, 1830 apresentem distribuição mundial, a maioria das infestações humanas são reportadas à América, África e Ásia (TAlari, 2004). Curiosamente, $L$. sericata pode ser utilizada em tratamentos de terapia larval visto que suas larvas também se alimentam de tecido necrótico (SHERman et al., 2000). Devido aos hábitos alimentares de suas larvas, estas moscas também são comuns em estudos forenses, pois participam ativamente da sucessão ecológica em cadáveres (Gomes \& Von Zuben, 2006).

Os dípteros califorídeos são os primeiros indivíduos a compor a sucessão faunística dos cadáveres humanos (Smith, 1986; Von Zuben, 2001), onde a estimativa da idade das larvas ou pupas mais velhas, coletadas no corpo bem como em sua circunvizinhança, irá indicar um intervalo pós-morte mínimo (Von Zuben, 2001). Entretanto, esta estimativa pode ser seriamente prejudicada pela não consideração da dispersão pósalimentar das larvas, podendo assim, comprometer as investigações médico-criminais (GoMES \& Von ZuBEN, 2004b).

Para a postura de seus ovos e alimentação de suas larvas, os califorídeos utilizam-se de substratos ricos em proteína (YAGHOOBI et al., 2005) onde as larvas procuram ingerir o máximo possível antes da exaustão dos recursos (UllyetT, 1950). As larvas dispersam em busca de novas fontes alimentares ou de um local para pupariação (GREENBERG, 1990), sendo que o local onde a pupa é encontrada indica a exata localização onde a larva parou de dispersar (Roux et al., 2006). Diante da importância do conhecimento pormenorizado dos aspectos relacionados à dispersão larval para os estudos em entomologia forense, o presente estudo objetivou simular o ambiente natural, em laboratório, a fim de verificar a propagação larval radial pós-alimentar de $L$. sericata e estabelecer relações entre as variáveis sexo, peso, distância e profundidade de enterramento das larvas na arena.

\section{MATERIAL E MÉTODOS}

Uma colônia préestabelecida e já adaptada de $L$. sericata (sexta geração) foi mantida durante todo o período de experimentação no Laboratório de Biologia de Insetos do Departamento de Microbiologia e 
Parasitologia da Universidade Federal de Pelotas (UFPel). Os insetos foram acondicionados em câmara climatizada com temperatura variando de $26^{\circ} \mathrm{C} \pm 2{ }^{\circ} \mathrm{C}$, umidade relativa do ar acima de $75 \%$ com fotofase de 12 horas. Os adultos foram criados em gaiolas teladas (dimensões 30 × 30 × 30 $\mathrm{cm}$ ) e alimentados com ração composta por açúcar refinado e farinha de carne em uma proporção de 2:1, além de água oferecida em copos Becker com espuma de poliestireno cobrindo a superfície do líquido.

Para postura dos mesmos utilizou-se um meio constituído por farinha de carne, serragem (3:1) e água, a fim de torná-lo pastoso. Junto a este meio, adicionou-se $50 \mathrm{~g}$ de fígado de frango a fim de proporcionar a maturidade reprodutiva das fêmeas. As posturas obtidas das gaiolas foram levadas a funis de alumínio, contendo o mesmo meio para postura, onde as larvas, após atingirem o contingente nutricional suficiente à pupariação, abandonaram caindo em um recipiente de coleta.

Dos recipientes de coleta dos funis foram retiradas, aleatoriamente, 350 larvas em condição pós-alimentar, que foram colocadas no centro de uma arena circular radial, dividida em quatro quadrantes possuindo $1 \mathrm{~m}$ de diâmetro e serragem úmida até atingir $10 \mathrm{~cm}$ de profundidade. As pupas foram localizadas quatro dias após a soltura e, com auxílio de réguas e trenas, sua profundidade e deslocamento foram avaliados. Estas foram individualizadas em frascos e, após 48 horas, pesadas em balança analítica. Após a emergência, houve a sexagem dos adultos. Durante todo o experimento, a arena foi mantida em câmara climatizada com temperatura variando de $26^{\circ} \mathrm{C} \pm 2{ }^{\circ} \mathrm{C}$, umidade relativa do ar acima de $75 \%$ com fotofase de 12 horas e iluminação homogênea a fim de evitar-se tendências de dispersão das larvas. Para este estudo de propagação larval radial pós-alimentar, foram utilizadas duas repetições. Os resultados obtidos referentes ao sexo, peso, distância percorrida para propagação e aprofundamento das larvas foram testados com qui-quadrado $\left(\chi^{2}\right)$, considerados estatisticamente significativos com $\alpha=0,05$ e de regressão a $95 \%$ de confiança.

\section{RESULTADOS}

Somando-se as duas repetições, foram recuperadas 567 pupas de L. sericata que foram localizadas, pesadas e sexadas. Embora tenha sido verificado que 27,69\% das larvas tenderam a se deslocar para o terceiro quadrante, o experimento permitiu observar que a arena circular possibilitou o deslocamento das larvas em todas as direções. Já para os outros quadrantes foram recuperadas $23,19 \%, 24,26 \%, 24,86 \%$ para o primeiro, segundo e quarto quadrante, respectivamente. Os valores referentes à distância percorrida, profundidade e peso das pupas em cada quadrante, estão representados na tabela I.

Quanto a profundidade média de enterramento das larvas, verificou-se que 255 larvas (45\%) puparam a uma profundidade compreendida entre $6 \mathrm{~cm}$ e $8 \mathrm{~cm}$ (Figs 1, 2). Do total, 14 larvas atingiram a profundidade máxima de enterramento $(10 \mathrm{~cm})$. Não observou-se diferenças significativas quanto a profundidade de enterramento de machos e fêmeas (Tab. II), havendo confirmação pelo teste $\left(\chi^{2}=0,055 ;\right.$ g.l. $\left.=3 ; \mathrm{p}>0,05\right)$. O mesmo foi observado quando comparada a profundidade de enterramento entre indivíduos viáveis (machos e fêmeas) e inviáveis $\left(\chi^{2}=4,31\right.$; g.l.=3; p>0,05) (Tab. II).

Em relação à distância percorrida do centro da arena, verificou-se uma grande variação, havendo uma maior concentração de pupas nas distâncias compreendidas entre $33 \mathrm{~cm}$ e $45 \mathrm{~cm}$ (Figs 1,2), sendo que somente duas larvas atingiram a distância máxima de deslocamento $(50 \mathrm{~cm})$. Quanto à distância percorrida pelos machos e pelas fêmeas, também não se observou diferença significativa (Tab. III) $\left(\chi^{2}=1,28 ;\right.$ g.1. $\left.=3 ; p>0,05\right)$. $\mathrm{O}$ mesmo foi observado quando comparada a distância percorrida entre indivíduos viáveis (machos e fêmeas) e inviáveis $\left(\chi^{2}=0,79 ;\right.$ g.l. $\left.=3 ; \mathrm{p}>0,05\right)$ (Tab. III).

A distribuição da média do peso das pupas pode ser observada na figura 3. A análise de correlação entre peso e distância percorrida $(r=0,246)$ e entre peso e profundidade $(\mathrm{r}=0,321)$ foi positiva. Resultados semelhantes foram obtidos por meio do teste de regressão entre peso e distância percorrida $(\mathrm{r}=0,607)$ e entre peso e profundidade $(\mathrm{r}=0,103)$, ou seja, as larvas mais pesadas dispersaram e se aprofundaram mais.

Não se verificou diferença significativa entre o peso dos indivíduos viáveis (machos e fêmeas) e dos inviáveis (Tab. IV) $\left(\chi^{2}=2,12 ;\right.$ g.l. $\left.=3 ; p>0,05\right)$. Ao comparar-se o peso dos indivíduos viáveis, observou-se que a média das pupas que originaram fêmeas ( $\mathrm{x}=32,35 \mathrm{mg})$ foi superior a das pupas que originaram machos $(x=30,28 \mathrm{mg})$ apresentando valores não significantes $\left(\chi^{2}=10,88\right.$; g.1. $=3$; $\mathrm{p}<0,05)$.

Foram calculados a média e o desvio-padrão da distância, profundidade e peso dos indivíduos machos, fêmeas e daqueles que foram inviáveis nas duas réplicas. As pupas inviáveis alcançaram médias de profundidade $(\mathrm{x}=5,23 \mathrm{~cm})$ e distância $(\mathrm{x}=27,11 \mathrm{~cm})$ menores quando comparadas com as pupas viáveis (Tab. V).
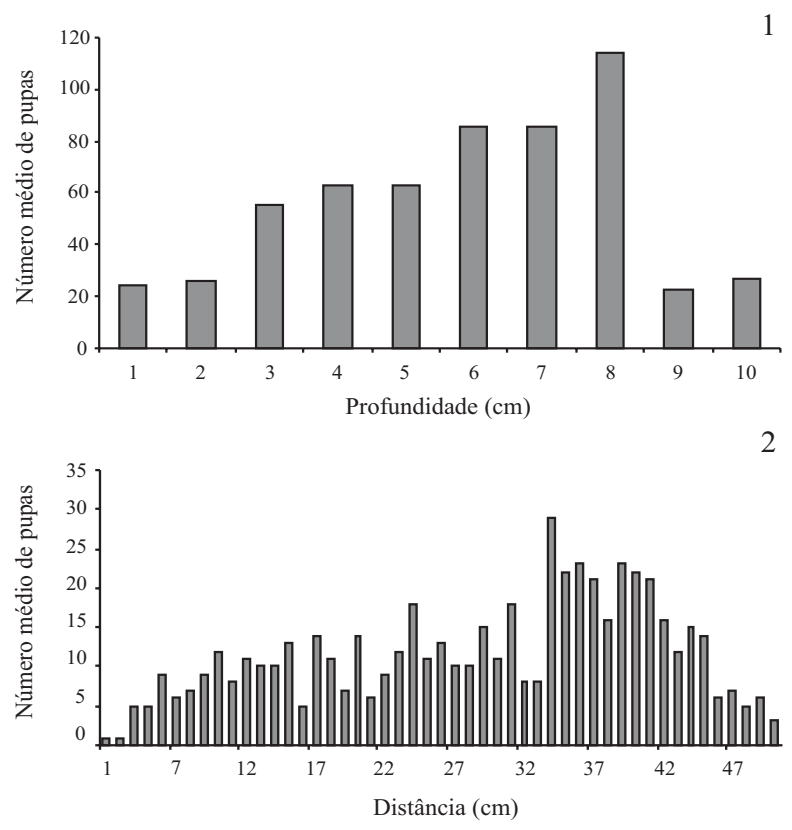

Figuras 1, 2. Distribuição de frequência dos valores de profundidade e distância de enterramento em que foram recuperadas as pupas de Lucilia sericata (Meigen, 1826) na arena circular, em condições de laboratório: 1, profundidade; 2, distância. 
Tabela I. Avaliação da propagação de larvas pós-alimentar de Lucilia sericata (Meigen, 1826), em arena circular, em condições de laboratório. Os números entre parênteses representam os valores mínimo-máximo encontrados para as diferentes variáveis.

\begin{tabular}{lcccc}
\hline Variáveis avaliadas & \multicolumn{3}{c}{ Quadrantes $(\%)$} \\
\cline { 2 - 5 } & 1 & 2 & 3 & 4 \\
\hline Média da distância $(\mathrm{cm})$ & $26,99(1-46)$ & $29,31(3-43)$ & $28,28(1-45)$ & $30,36(2-50)$ \\
Desvio padrão da distância $( \pm)$ & 5,57 & 11,54 & 12,69 & 10,92 \\
Média da profundidade $(\mathrm{cm})$ & $6,39(0,3-9)$ & $5,54(0,5-9,8)$ & $5,01(0,5-10)$ & $5,72(0,8-10)$ \\
Desvio padrão da profundidade $( \pm)$ & 2,57 & 1,93 & 2,36 & 2,10 \\
Média do peso (mg) & $31,52(22-35)$ & $31,52(20-38)$ & $29,77(19-36)$ & $31,87(20-35)$ \\
Desvio padrão do peso $(\mathrm{mg})( \pm)$ & 0,81 & 0,80 & 0,77 & 0,70
\end{tabular}

Tabela II. Relação entre a profundidade de enterramento, sexo e viabilidade das larvas de Lucilia sericata (Meigen, 1826) em arena circular, em condições de laboratório. Qui-quadrado entre os sexos em relação a profundidade de enterramento sem diferenças significativas: $\chi^{2}=0,055 ;$ g.l. $=3 ; \mathrm{p}>0,05$; assim como para indivíduos viáveis e inviáveis foi $\chi^{2}=4,31 ; \mathrm{g} .1 .=3 ; \mathrm{p}>0,05$ (Abs, absoluta; F, frequência; Prof, profundidade; Rel, relativa).

\begin{tabular}{lcccccccc}
\hline Prof $(\mathrm{cm})$ & \multicolumn{2}{c}{ Machos } & \multicolumn{2}{c}{ Fêmeas } & \multicolumn{2}{c}{ Inviáveis } & \multicolumn{2}{c}{ Total Frequência } \\
\cline { 2 - 9 } & F. Abs $(\mathrm{n})$ & F. Rel (\%) & F. Abs (n) & F. Rel (\%) & F. Abs (n) & F. Rel (\%) & Abs (n) & Rel (\%) \\
\hline$\leq 5$ & 74 & 13,05 & 72 & 12,70 & 85 & 14,99 & 231 & 40,74 \\
$>5$ & 119 & 20,99 & 121 & 21,34 & 96 & 16,93 & 336 & 59,26 \\
Total & 193 & 34,04 & 193 & 34,04 & 181 & 31,92 & 567 & 100,00 \\
\hline
\end{tabular}

Tabela III. Relação entre distância percorrida, sexo e viabilidade das larvas de Lucilia sericata (Meigen, 1826) em arena circular, em condições de laboratório. Qui-quadrado entre os sexos em relação a distância percorrida sem diferenças significativas: $\chi^{2}=1,28$; g.l. $=3$; p>0,05; assim como para indivíduos viáveis e inviáveis foi $\chi^{2}=0,79$; g.l. $=3$; p>0,05 (Abs, absoluta; Dist, distância; F, frequência; Rel, relativa).

\begin{tabular}{lcccccccc}
\hline Dist $(\mathrm{cm})$ & \multicolumn{2}{c}{ Machos } & \multicolumn{2}{c}{ Fêmeas } & \multicolumn{2}{c}{ Inviáveis } & \multicolumn{2}{c}{ Total Frequência } \\
\cline { 2 - 8 } & F. Abs (n) & F. Rel (\%) & F. Abs (n) & F. Rel (\%) & F. Abs (n) & F. Rel (\%) & Abs (n) & Rel (\%) \\
\hline$\leq 30$ & 85 & 14,99 & 96 & 16,93 & 92 & 16,22 & 273 & 48,15 \\
$>30$ & 108 & 19,05 & 97 & 17,11 & 89 & 15,70 & 294 & 51,85 \\
Total & 193 & 34,04 & 193 & 34,04 & 181 & 31,92 & 567 & 100,00 \\
\hline
\end{tabular}

Tabela IV. Relação do peso, sexo e viabilidade das pupas de Lucilia sericata (Meigen, 1826) que foram recuperadas em arena circular, em condições de laboratório. Qui-quadrado entre os sexos em relação ao peso se observou diferenças significativas: $\chi^{2}=10,88 ; \mathrm{g} .1 .=3 ; \mathrm{p}<0,05$; já em relação aos indivíduos viáveis e inviáveis sem diferenças significativas $\chi^{2}=2,12$; g.1.=3; p>0,05 (Abs, absoluta; F, frequência; Rel, relativa).

\begin{tabular}{lcccccccc}
\hline Peso (mg) & \multicolumn{2}{c}{ Machos } & \multicolumn{2}{c}{ Fêmeas } & \multicolumn{2}{c}{ Inviáveis } & \multicolumn{2}{c}{ Total Frequência } \\
\cline { 2 - 9 } & F. Abs (n) & F. Rel (\%) & F. Abs (n) & F. Rel (\%) & F. Abs (n) & F. Rel (\%) & Abs (n) & Rel (\%) \\
\hline$\leq 35$ & 140 & 24,70 & 109 & 19,22 & 128 & 22,57 & 377 & 66,49 \\
$>35$ & 53 & 9,35 & 84 & 14,81 & 53 & 9,35 & 190 & 33,51 \\
Total & 193 & 34,05 & 193 & 34,03 & 181 & 31,92 & 567 & 100,00 \\
\hline
\end{tabular}

Tabela V. Avaliação da propagação de machos e fêmeas de Lucilia sericata (Meigen, 1826) distribuídas nos dois experimentos realizados para estudo de dispersão larval radial pós-alimentar (Dist, distância; Inv, inviáveis; Prof, profundidade).

\begin{tabular}{ccccc}
\hline Variáveis Avaliadas & Sexo & Experimento $1 \times \pm$ DP $(\mathrm{N})$ & Experimento $2 \times \pm$ DP $(\mathrm{N})$ & Média Total $\mathrm{x} \pm$ DP $(\mathrm{N})$ \\
\hline Prof $(\mathrm{cm})$ & Inv & $4,45 \pm 2,49(89)$ & $5,96 \pm 2,29(93)$ & $5,23 \pm 2,50(182)$ \\
& Fêmea & $4,94 \pm 2,24(103)$ & $6,82 \pm 1,82(90)$ & $5,82 \pm 2,25(193)$ \\
& Macho & $5,06 \pm 2,12(94)$ & $6,63 \pm 1,79(98)$ & $5,86 \pm 2,10(192)$ \\
& Média & $4,82 \pm 2,28(286)$ & $6,47 \pm 1,97(281)$ & $5,71 \pm 2,26(567)$ \\
Dist $(\mathrm{cm})$ & Inv & $26,02 \pm 12,74(89)$ & $28,14 \pm 13,15(93)$ & $27,11 \pm 12,96(182)$ \\
& Fêmea & $24,37 \pm 11,95(103)$ & $34,37 \pm 9,37(90)$ & $29,03 \pm 11,90(193)$ \\
& Macho & $27,74 \pm 12,16(94)$ & $32,18 \pm 9,91(98)$ & $29,99 \pm 11,27(192)$ \\
Peso $(\mathrm{mg})$ & Média & $26,04 \pm 12,28(286)$ & $31,56 \pm 10,81(281)$ & $29,18 \pm 11,81(567)$ \\
& Inv & $26,52 \pm 7,03(89)$ & $34,81 \pm 5,89(93)$ & $30,67 \pm 7,70(182)$ \\
& Fêmea & $26,70 \pm 6,08(103)$ & $38,81 \pm 4,11(90)$ & $32,35 \pm 8,01(193)$ \\
& Macho & $25,56 \pm 7,46(94)$ & $34,86 \pm 3,14(98)$ & $30,28 \pm 7,35(192)$ \\
& Média & $26,26 \pm 6,86(286)$ & $36,16 \pm 4,38(281)$ & $31,13 \pm 7,72(567)$
\end{tabular}




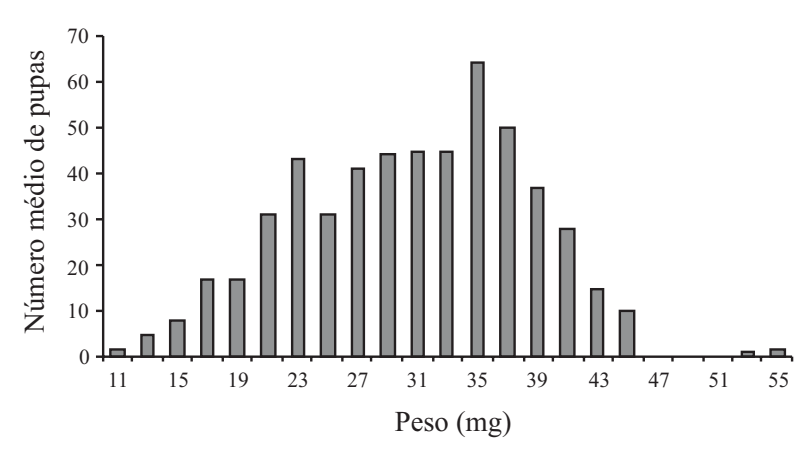

Figura 3. Distribuição da média de peso das pupas de Lucilia sericata (Meigen, 1826) recuperadas na arena circular, em condições de laboratório.

\section{DISCUSSÃO}

Aparentemente a profundidade da arena utilizada no presente estudo pode ter limitado o deslocamento vertical das larvas de $L$. sericata pois, em experimento semelhante, utilizando o mesmo substrato de pupariação, realizado com Lucilia cuprina (Wiedmann, 1830) a maioria das pupas foi encontrada a uma profundidade média de $14,58 \mathrm{~cm}$ (Gomes \& Von Zuben, 2004a). Trabalhos realizados com outros dípteros, referentes a propagação em arena circular, utilizando serragem como substrato de pupariação (Gomes et al., 2003; GOMEs \& Von ZuBEN, 2006), demonstraram grande variação quanto à profundidade média de pupariação. Segundo Gomes \& Von Zuben (2004b), as larvas tendem a se aprofundar mais ou menos de acordo com a temperatura à qual estão submetidas, havendo um maior enterramento na tentativa de escapar das temperaturas mais baixas.

Gomes \& Von Zuben (2004a) verificaram que Lucilia cuprina se concentra entre $15 \mathrm{~cm}$ e $20 \mathrm{~cm}$ do local de origem. Estes resultados demonstraram que L. sericata se aprofunda menos, porém dispersa mais em relação ao centro da arena quando comparada com L. cuprina. As diferenças encontradas entre estas duas espécies, provavelmente, se devem às características específicas, considerando que o peso influenciou de maneira diferente nas duas espécies.

Segundo BoldRINI et al. (1997), essa variação na frequência de pupas em função da distância seria uma consequência da formação de agregações larvais em determinado local do substrato de pupariação. As larvas dispersantes seriam capazes de perceber a densidade de pupas já enterradas em um determinado ponto do substrato, sendo que aglomerações num determinado ponto, induziriam larvas em movimento ao buscarem outros sítios de pupariação mais distantes. O comportamento de dispersão larval pós-alimentar e o consequente padrão de distribuição espacial das pupas nos sítios de pupariação, além de objetivarem busca de recursos alimentares, podem ter implicações na maior susceptibilidade das mesmas ao ataque de predadores e parasitóides em populações de ambientes naturais, bem como fuga das adversidades bióticas e abióticas (LEGNER, 1977; PESCHKE et al., 1987; BOLDRINI et al, 1997; GOMES \& Von Zuben, 2004a).

De acordo com os resultados referente ao peso das pupas e a distância percorrida pelas larvas, observaram-se que as larvas mais pesadas dispersaram e se aprofundaram mais, não conferindo com estudos semelhantes realizados por outros autores (GOMEs \& VON Zuben, 2002; Gomes \& Von Zuben, 2004a). Segundo Gomes et al. (2003), as pupas mais leves e que pouco dispersaram, provavelmente, tenham atingido distância e profundidade máximas e voltado ao centro da arena conferindo correlação positiva entre peso, distância e profundidade.

Estes estudos de dispersão larval pós-alimentar têm importantes implicações em investigações médicocriminais, pois a presença de imaturos em cadáveres humanos ou em suas circunvizinhanças atua no auxílio da estimativa do tempo entre a morte e a descoberta do cadáver (Gomes \& Von Zuben, 2002). Esta estimativa constitui um dos aspectos fundamentais para a medicina legal (Sмітн, 1986), sendo que a mesma pode ser prejudicada pela não consideração da dispersão pósalimentar (Von ZuBEN, 1998).

Com este experimento conclui-se que em uma arena circular em condições de laboratório, as larvas de $L$. sericata propagam em todas as direções. Em L. sericata não houve aglomerações pupais em nenhum dos quadrantes analisados. A distância de propagação e a profundidade de pupariação são influenciadas pelo tamanho das larvas, isso foi constatado levando em consideração o peso das pupas encontradas. Já o sexo não causa influência no deslocamento e enterramento das larvas.

\section{REFERÊNCIAS BIBLIOGRÁFICAS}

Boldrini, J. L.; Bassanezi, R. C.; Moretti, A. C.; Von Zuben, F. J.; Godoy, W. A. C.; Von Zuben, C. J. \& Reis, S. F. 1997. Non-local interactions and the dynamics of dispersal in immature insects. Journal of Theoretical Biology 185:523-531.

Daniel, M.; Sramova, H. \& ZalabSKA, E. 1994. Lucilia sericata (Diptera: Calliphoridae) causing hospital-acquired myiasis of a traumatic wound. Journal of Hospital Infection 28:149152 .

Gomes, L. \& Von Zuben, C. J. 2002. Dispersão larval pós-alimentar em Chrysomya megacephala (F.) (Diptera: Calliphoridae): Profundidade, distância e peso de enterramento para pupariação. Bioscience Journal 18:67-76.

2004a. Dispersão larval radial pós-alimentar em Lucilia cuprina (Diptera, Calliphoridae): profundidade, peso e distância de enterramento para pupação. Iheringia, Série Zoologia, 94(2): 135-138.

2004b. Efeito da temperatura na profundidade de enterramento de larvas de Chrysomya megacephala (Fabricius, 1794) (Diptera: Calliphoridae) sob condições controladas. Entomología y Vectores 11(3):551-557.

2006. Forensic entomology and main challenges in Brazil. Neotropical Entomology 35(1):1-11.

Gomes, L.; Von Zuben, C. J. \& Sanches, M. R. 2003. Estudo da dispersão larval radial pós-alimentar em Chrysomya megacephala (Fabricius, 1794) (Diptera, Calliphoridae). Revista Brasileira de Entomologia 47(2):229-234.

Grassberger, M. \& Reiter, C. 2001. Effect of temperature on Lucilia sericata (Diptera: Calliphoridae) development with special reference to the isomegalen-and isomorphen-diagram. Forensic Science International 120:32-36.

GreenberG, B. 1990. Behavior of postfeeding larvae of some Calliphoridae and a muscid (Diptera). Annals of the Entomological Society of America 83:1210-1214.

LeGNER, E. F. 1977. Temperature, humidity and depth of habitat influencing host destruction and fecundity of muscoid fly parasites. Entomophaga 22:199-206. 
Peschke, K.; Krapf, D. \& Fuldner, D. 1987. Ecological separation, functional relationships, and limiting resources in a carrion insect community. Zoologischer Jahresbericht 114:241265.

Roux, O.; Gers, C.; Telmon, N. \& Legal, L. 2006. Circular dispersal of larvae in the necrophagous Diptera Protophormia terraenovae (Diptera: Calliphoridae). Annales de la Societe Entomologique de France 42(1):51-56.

Sherman, R. A.; Hall, M. J. R. \& Thomas, S. 2000. Medicinal maggots: an ancient remedy for some contemporary afflictions. Annual Review of Entomology 45:55-81.

Sмітн, K. G. V. A. 1986. Manual of Forensic Entomology. Ithaca, Cornell University. 205p.

Talari, S. A.; Sadr, F.; Doroodgar, A.; TAlari, M. R. \& Gharabagh,
A. S. 2004. Wound myiasis by Lucilia sericata. Archives Iranian Medicine 7(2):128,129

Ullyett, G. C. V. 1950. Competition for food and allied phenomena in sheep blowfly populations. Philosophical Transactions of the Royal Society of London 234:77-174.

Von ZuBen, C. J. 1998. Comportamento de oviposturas individuais, percentagem de eclosão e peso larval mínimo para pupação em populações de Chrysomya megacephala (F.). Anais da Sociedade Entomologica do Brasil 4:525-533.

2001. Zoologia aplicada: recentes avanços em estudos de entomologia forense. Entomología y Vectores 8(2):173-183.

YAghoobi, R.; TiRgari, S. \& SinA, N. 2005. Human auricular myiasis caused by Lucilia sericata: clinical and parasitological considerations. Acta Medica Iranica 43(2):155-157. 